\title{
Subacute massive pulmonary embolism treated with plasminogen and streptokinase
}

\author{
DA ELLIS, E NEVILLE, RJC HALL \\ From the Royal Victoria Infirmary and Freeman Hospital, Newcastle upon Tyne
}

ABSTRACT Major pulmonary embolism occurring insidiously over several weeks (subacute massive pulmonary embolism) has a high mortality and may not respond well to standard anticoagulant or thrombolytic treatment. A priming dose of plasminogen was used to enhance thrombolysis produced by a streptokinase infusion in five consecutive patients with subacute massive pulmonary embolism. In each patient a dramatic clinical improvement occurred with a substantial increase in pulmonary blood flow. All five patients survived to leave hospital. Malignant disease was the underlying cause of embolism in three patients, two of whom died of their malignant disease in the six months after treatment of their pulmonary embolism. The third patient with malignant disease had a choriocarcinoma; at least some of the pulmonary obstruction may have been tumour tissue but this obstruction was dramatically cleared by the treatment. The use of a combination of plasminogen with streptokinase should be considered in severely ill patients with subacute massive pulmonary embolism, particularly if other treatment, including streptokinase alone, has failed.

Subacute massive pulmonary embolism has been clearly defined by Sutton et al. ${ }^{1}$ Patients have a history of progressive breathlessness of at least two weeks' duration, without an episode of cardiovascular collapse, and pulmonary angiography shows obstruction of at least half of the pulmonary circulation. Breathlessness is characteristically insidious in onset, chest pain and haemoptysis may or may not occur, and the findings on examination usually suggest right heart strain. Although it is an uncommon condition subacute massive pulmonary embolism has a high early mortality rate and the response to treatment with heparin or streptokinase may be disappointing. ${ }^{2}$ The reason for the poor response to treatment may lie in the age of the thrombus; the plasminogen content of established thrombus falls with time ${ }^{3}$ and plasminogen is the major substrate on which streptokinase acts to produce clot lysis. Improved lysis of old thrombus has been shown in peripheral veins by the infusion of plasminogen followed by streptokinase ${ }^{4}$ and in acute pulmonary embolism by the use of a combination of plasminogen and urokinase. ${ }^{5}$ We have used a similar

Address for reprint requests: Dr RJC Hall, Royal Victoria Infirmary, Newcastle upon Tyne NE1 4LP.

Accepted 18 August 1983 approach in patients with subacute massive pulmonary embolism and our preliminary findings are reported here.

\section{Patients and methods}

In five consecutive patients in whom the clinical features suggested subacute massive pulmonary embolism the diagnosis was confirmed by pulmonary angiography and pulmonary artery pressures were measured with standard electronic pressure transducers. Cardiac output was not measured. Angiograms were reported according to a numerical scoring system $^{6}$ to define the extent to which blood vessels were affected (score 0-16) and regional blood flow was reduced (score $0-18$ ). The scores for vascular involvement and flow were added. A score of 0 represents a normal pulmonary angiogram whereas a theoretical score of 34 would represent complete obstruction of pulmonary flow with all pulmonary arteries affected by clot. A score of 17 or greater (that is, $>50 \%$ ) represents "massive" pulmonary embolism.

Each patient received human placental plasminogen (lys plasminogen) $150 \mathrm{mg}$ in $200 \mathrm{ml}$ normal saline by infusion over 30 minutes through a Swan Ganz or 8F NIH catheter in the main pulmonary artery. An infusion of streptokinase was begun 
Pulmonary artery pressures and angiographic scores before and after treatment with plasminogen and streptokinase

\begin{tabular}{|c|c|c|c|c|c|c|c|c|c|c|c|c|c|c|}
\hline \multirow{3}{*}{ Patient } & \multirow{3}{*}{$\begin{array}{c}\text { Age } \\
\text { (y) } \\
\text { Sex }\end{array}$} & \multicolumn{5}{|c|}{ Before treatment } & \multicolumn{4}{|c|}{ After treatment $(24 h)$} & \multicolumn{4}{|l|}{ Late† } \\
\hline & & \multirow{2}{*}{$\begin{array}{l}\text { Duration } \\
\text { of } \\
\text { dyspnoea } \\
\text { (months) }\end{array}$} & \multirow{2}{*}{$\begin{array}{l}P A \\
\text { pressure } \\
(\mathrm{mm} \mathrm{Hg})\end{array}$} & \multicolumn{3}{|c|}{ Angiographic score* } & \multirow{2}{*}{$\begin{array}{l}P A \\
\text { pressure } \\
\text { (mm } \mathrm{Hg})\end{array}$} & \multicolumn{3}{|c|}{ Angiographic score* } & \multirow{2}{*}{$\begin{array}{l}P A \\
\text { pressure } \\
(\mathrm{mm} \mathrm{Hg})\end{array}$} & \multicolumn{3}{|c|}{ Angiographic score* } \\
\hline & & & & $\begin{array}{l}\text { Vessels } \\
\text { affected }\end{array}$ & Flow & Total & & $\begin{array}{l}\text { Vessels } \\
\text { affected }\end{array}$ & Flow & Total & & $\begin{array}{l}\text { Vessels } \\
\text { affected }\end{array}$ & Flow & Total \\
\hline 1 & $\begin{array}{l}44 \\
\mathrm{M}\end{array}$ & 1 & $70 / 25$ & 16 & 11 & 27 & $42 / 16$ & 15 & 7 & 22 & $29 / 8$ & 7 & 2 & 9 \\
\hline 2 & $\begin{array}{l}48 \\
M\end{array}$ & 4 & $50 / 16$ & 13 & 9 & 22 & - & 13 & 4 & 17 & $30 / 8$ & 11 & 2 & 13 \\
\hline 3 & $\begin{array}{l}65 \\
M\end{array}$ & 1 & $80 / 30$ & 16 & 13 & 29 & $60 / 20$ & 14 & 7 & 21 & $35 / 15$ & 3 & 2 & 5 \\
\hline 4 & $\begin{array}{l}58 \\
\mathrm{M}\end{array}$ & 2 & $80 / 40$ & 16 & 9 & 25 & $40 / 12$ & 16 & 5 & 21 & $35 / 15$ & 15 & 5 & ; 20 \\
\hline 5 & $\begin{array}{l}31 \\
\mathrm{~F}\end{array}$ & 3 & $44 / 14$ & $\begin{array}{l}14 \\
(9)^{*}\end{array}$ & $\begin{array}{l}10 \\
(8)\end{array}$ & $\begin{array}{c}24 \\
(17)\end{array}$ & - & $\overline{(9)}$ & $\overline{(6)}$ & $\overline{(15)}$ & - & $\overline{(6)}$ & $\overline{(5)}$ & $\overline{(11)}$ \\
\hline
\end{tabular}

*Figures in parentheses are for right lung scores only. The scoring system used scores normal pulmonary arteries and no embolism as 0 and complete obstruction of all vessels as 34 (vessels affected out of 16 and flow out of 18 ). The higher the figure for vessels affected the greater the number of vessels affected but the higher the figure for flow the lower the flow.

$\dagger$ Three days in patient 2 , five days in patient 5 , two months in patients 1,3 and 4 .

PA-pulmonary artery.

immediately afterwards through the same catheter. A loading dose of 600000 units in $100 \mathrm{ml} \mathrm{5 \%}$ dextrose was followed by a continuous infusion of 100000 units in $50 \mathrm{ml} \mathrm{5 \%}$ dextrose hourly for 24 hours. A bolus of hydrocortisone $100 \mathrm{mg}$ intravenously every six hours was given during the infusion to minimise reactions to streptokinase. Pulmonary artery pressure measurements and angiography were repeated at the end of the streptokinase infusion and a decision was made on the need for further thrombolytic treatment. In two patients the same sequence of plasminogen followed by streptokinase was repeated. Thereafter heparin was given by continuous infusion until the patient was fully mobile and this was followed by long term anticoagulation with warfarin.
The patients were aged $31-65$ years; only one was female. All presented with progressive breathlessness of one to four months' duration. Each patient had a tachycardia with hypotension, poor peripheral circulation, and a gallop rhythm on auscultation. Electrocardiograms showed features of right heart strain in each case. No known predisposing factors were evident at the time of presentation but a diagnosis of malignant neoplasm was subsequently made in three patients. The first four patients were critically ill at the time of diagnosis and received plasminogen and streptokinase as initial treatment. Patient 5 received plasminogen and streptokinase after clinical deterioration during anticoagulant treatment with heparin followed by warfarin.

\section{Vessels affected}

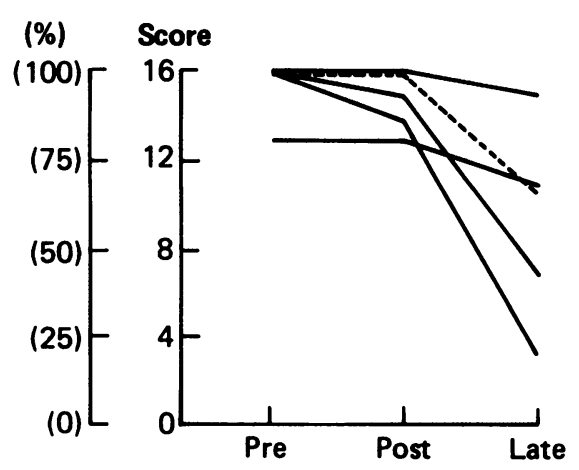

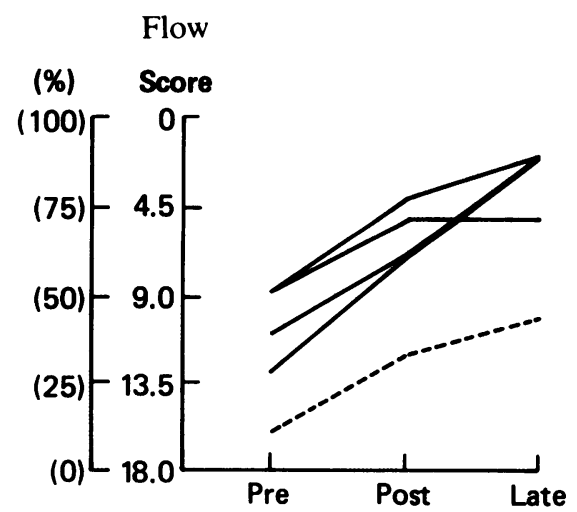

Fig 1 Pulmonary arteries affected by thrombus and pulmonary blood flow before and after treatment with plasminogen and streptokinase. Improvement in blood flow occurs early with a later reduction in the number of vessels affected by thrombus. Dotted lines are percentage scores for right lung angiograms only. 

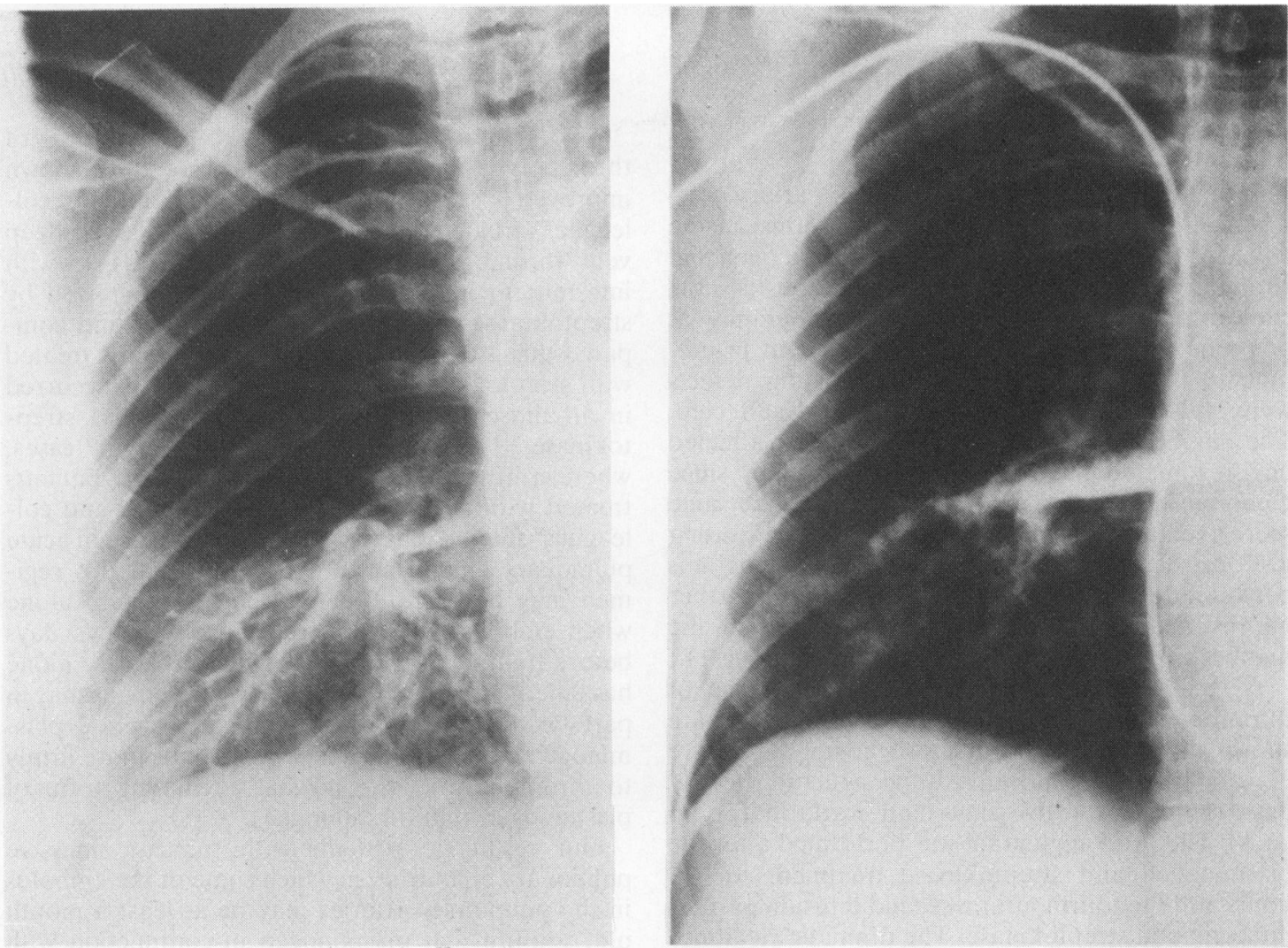

b
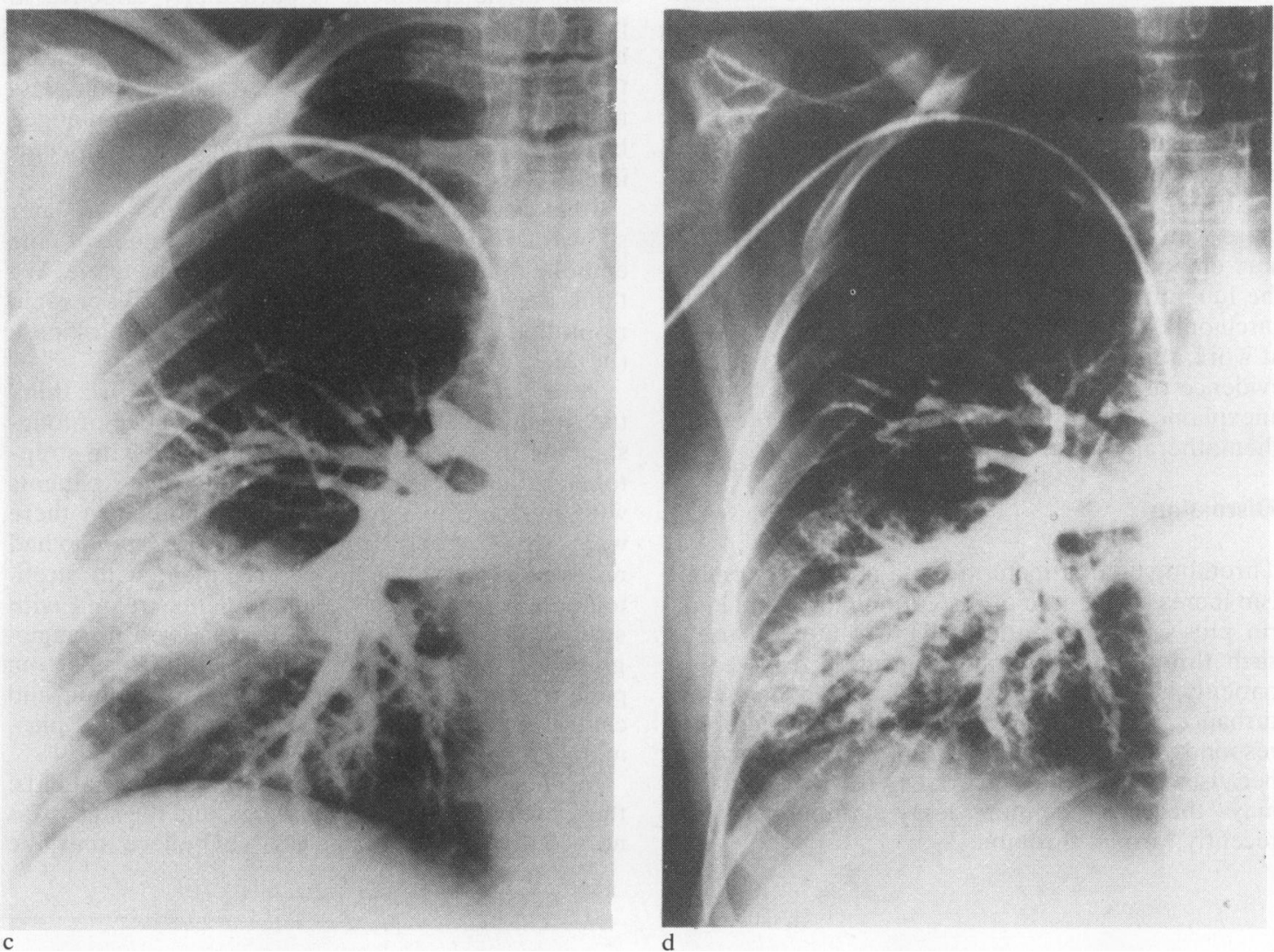

Fig 2 Right lung angiograms from case 5: $(a)$ and $(b)$ before and after anticoagulant treatment with heparin and warfarin and (c) and (d) after 24 hours' and 48 hours' treatment with plasminogen and streptokinase. 


\section{Results}

The angiographic scores and pulmonary artery pressures before and after treatment are shown in the table. At the end of treatment with plasminogen and streptokinase all five patients showed considerable clinical improvement. Pulmonary angiography at this time showed appreciable improvement in pulmonary blood flow, although some filling defects were still visible in the vessels previously affected. The angiographic score for blood vessels affected underestimated the degree of improvement since emboli in the same site continued to have the same score even though smaller in size and hence causing less obstruction. Subsequent pulmonary angiograms performed up to two months later showed a further improvement in blood flow and decrease in the number of vessels containing filling defects (fig 1).

As an example of the degree of clearance of thrombus the right lung angiograms of patient 5 are shown in figure 2 . The first two angiograms were performed before and after anticoagulation for 21 days (heparin for five days and warfarin for 16 days). The third angiogram was performed after the plasminogen and streptokinase treatment for 24 hours and the fourth after a second infusion of plasminogen and streptokinase. The dramatic clearance of thrombus is evident and this coincided with considerable clinical improvement, which has been maintained. Complications were limited to minor bleeding from venepuncture sites except in patient 5 , in whom substantial vaginal bleeding occurred. The investigation of this vaginal bleeding led to the diagnosis of choriocarcinoma.

All five patients survived to leave hospital but there were three late deaths not related to pulmonary embolism. Patient 2 died of adenocarcinoma of the lung after five months and patient 4 of colonic carcinoma after six months. Patient 1 died suddenly at work after 16 months. At necropsy there was no evidence of pulmonary embolism and this death is unexplained. Patient 5 is now receiving chemotherapy for choriocarcinoma.

\section{Discussion}

Thrombolytic treatment in acute pulmonary embolism increases the rate of resolution more than heparin plus coumarin anticoagulants ${ }^{67}$ but the use of such thrombolytic therapy is usually reserved for patients with large emboli and haemodynamic disturbance. Subacute massive pulmonary embolism responds less well to streptokinase, ${ }^{2}$ possibly because the embolic material is relatively old and may therefore contain less plasminogen than recently formed thrombus. ${ }^{3}$
Two important but uncontrolled studies of thrombolytic therapy and plasminogen have shown improved lysis of thrombus. Kakkar and his colleagues ${ }^{4}$ treated 12 patients with established deep vein thrombosis (mean duration 8.1 days) with intermittent infusions of plasminogen followed by streptokinase over a period of five days and compared this group with a further five patients treated with streptokinase alone. Substantial lysis occurred in all those treated with plasminogen and streptokinase, lysis being complete in eight cases, whereas little or no lysis occurred in the patients treated with streptokinase alone. Griguer and colleagues ${ }^{5}$ studied plasminogen and urokinase in acute pulmonary embolism and suggested that this regimen may be more effective than urokinase alone when embolism has occurred more than five days before treatment is begun or when urokinase alone has failed. The effectiveness of such regimens may in part be due to the use of human placental plasminogen (lys plasminogen), which binds more firmly to thrombus than the normal circulating form of plasminogen (glu plasminogen). ${ }^{8}$

Our results in patients with subacute massive pulmonary embolism, in which some of the embolus in the pulmonary arteries may be at least a month old, confirm that plasminogen in conjunction with thrombolytic treatment is particularly effective in producing lysis of older pulmonary emboli. This finding is in keeping with the observations that old thrombus contains only small quantities of plasminogen and that administered lys plasminogen binds firmly to thrombus, rendering it more susceptible to lysis by streptokinase.

It is possible that in patient 5, who was later shown to have choriocarcinoma, some of the embolic material may have been tumour tissue. We think that this is unlikely in view of the very rapid resolution seen after plasminogen and streptokinase (fig 2).

Although this study was uncontrolled we think that it is unlikely that such rapid clearance of longstanding thrombus would have occurred with streptokinase alone. In a previous study of 24 patients with subacute massive pulmonary embolism there were $9(38 \%)$ early deaths, four in patients who had received at least 24 hours' treatment with streptokinase. Of a further eight patients treated with streptokinase for 72 hours, two showed no angiographic improvement. ${ }^{2}$ In contrast, all five of our patients showed considerable haemodynamic and clinical improvement after treatment with plasminogen and streptokinase.

We have studied only a small number of patients; these are our preliminary findings, and the study was not controlled. Nevertheless we believe that the 
results are important since subacute massive pulmonary embolism, although uncommon, has a high mortality rate when treated by conventional means. A controlled study of plasminogen with streptokinase and streptokinase alone is now being undertaken but will not be complete for several years because of the rarity of such patients.

We suggest that, while better evidence is awaited, the use of plasminogen and streptokinase in combination should be considered in patients with subacute massive pulmonary embolism who have considerable haemodynamic disturbance and have failed to respond to heparin or streptokinase alone.

We are grateful to Dr F Toulemonde (Chouay Laboratories) and Kabi Pharmaceuticals for supplies of plasminogen.

\section{References}

' Sutton GC, Hall RJC, Kerr IH. Clinical course and later prognosis of treated subacute massive, acute minor and chronic pulmonary thromboembolism. Br Heart J 1977;39:1135-42.
${ }^{2}$ Hall RJC, McHaffie D, Pusey C, Sutton GC. Subacute massive pulmonary embolism. $\mathrm{Br}$ Heart $J$ 1981;45:681-8.

${ }^{3}$ Ogston D, Ogston CM, Fullerton HW. The plasminogen content of thrombi. Thromb Diath Haemorrh 1966;15:220-30.

${ }^{4}$ Kakkar VV, Sagar S, Lewis M. Treatment of deep-vein thrombosis with intermittent streptokinase and plasminogen infusion. Lancet 1975;ii:674-6.

${ }^{5}$ Griguer P, Charbonnier B, Latour F, Fauchier JP, Brochier M. Plasminogen and moderate doses of urokinase in the treatment of acute pulmonary embolism. Angiology 1979;30:1-12.

${ }^{6}$ Miller GAH, Sutton GC, Kerr IH, Gibson RV, Honey M. Comparison of streptokinase and heparin in treatment of isolated acute massive pulmonary embolism. Br Med J 1971;ii:681-4.

${ }^{7}$ Urokinase Pulmonary Embolism Trial Study Group. Urokinase pulmonary embolism trial: phase 1 results. JAMA 1970;214:2163-72.

${ }^{8}$ Thorsen S. Differences in the binding to fibrin of native plasminogen and plasminogen modified by proteolytic degradation: influence of $\omega$-aminocarboxylic acids. Biochem Biophys Acta 1975;393:55-65. 